\title{
Restoring with Flowables
}

Authors: Douglas A. Terry

Publisher: Quintessence Publishing Co Inc

Language: English

ISSN: 978-0-86715-668-3

Edition: 1/e

Publish Year: 2017

Pages: 292, illustrated

Price: $\$ 148.00$

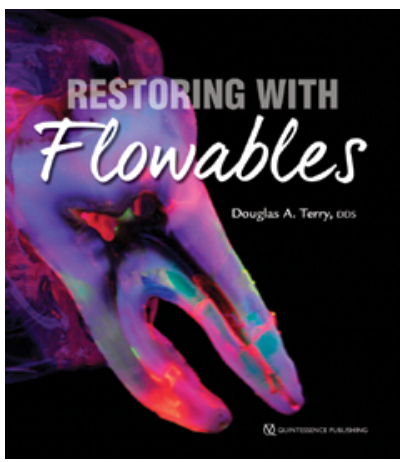

The book entitled "Restoring with Flowables" by Dr. Douglas A. Terry presents innovative concepts in restorative dentistry. He shows the applications of next-generation flowable composites in a step-by-step manner.

This book has four chapters, starting with the historical perspective and the evolution of flowable composites. Then it approaches the general consideration of adhesion, and tooth preparation. According to aesthetic principles, in the last two chapters, the author talks about the clinical applications of flowable direct restorations in anterior and posterior teeth; sealants and preventive resin restorations; provisional fabrication, modification, and repair; tooth splinting; eliminating cervical tooth sensitivity; adhesive reattachments of tooth fragment; developing the ovate pontic site; bonding porcelain veneers and inlay cementation; stratification layering technique; restoration of anterior and posterior primary tooth; developing a post and core; and restoring form and function, among others. The book provides detailed and very well illustrated protocols of the adhesive design concept, evolution offlowable resin composites and applications of contemporary restorative dentistry. Whether you are an experienced clinician or a young dentist this book will give you a new perspective of minimally invasive and aesthetic restorations.

Marian-Vladimir Constantinescu

$\mathrm{DDS}, \mathrm{PhD}$

Holistic Dental \& Medical Institute of Bucharest-ROPOSTURO, Bucharest

Romania

e-mail:

dr.vladimir.constantinescu@gmail.com 